\title{
A note on bimodal singularities and mirror symmetry
}

\author{
Makiko Mase and Kazushi Ueda
}

\begin{abstract}
We discuss the relation between transposition mirror symmetry of Berlund and Hübsch for bimodal singularities and polar duality of Batyrev for associated toric K3 hypersurfaces. We also show that homological mirror symmetry for singularities implies the geometric construction of Coxeter-Dynkin diagrams of bimodal singularities by Ebeling and Ploog.
\end{abstract}

\section{Introduction}

Mirror symmetry is a mysterious relationship between symplectic geometry and complex geometry motivated by string theory. It was originally discovered in the context of CalabiYau manifolds, but it has soon become clear that singularity theory is an important aspect of the theory through the Calabi-Yau/Landau-Ginzburg correspondence [VW89, GVW89, Mar90, Wit93.

In this paper, we discuss the relation between mirror symmetry for bimodal singularities and mirror symmetry for K3 surfaces. Bimodal singularities are a natural class of singularities which come next to simple (or 0-modal) singularities and unimodal singularities. They are classified by Arnold [Arn75, Arn76] into 8 infinite series and 14 exceptional families. We will deal only with singularities defined by invertible polynomials, since we use transposition mirror construction of Berglund and Hübsch [BH93], which makes sense only for invertible polynomials. An invertible polynomial is a polynomial of the form

$$
f\left(x_{1}, \ldots, x_{n}\right)=f_{A}\left(x_{1}, \ldots, x_{n}\right)=\sum_{i=1}^{n} x_{1}^{a_{i 1}} \cdots x_{n}^{a_{i n}}
$$

having an isolated singularity at the origin. Here $A=\left(a_{i j}\right)_{i, j=1}^{n}$ is an $n \times n$ matrix with positive integer entries. Its Berglund-Hübsch transpose is the invertible polynomial

$$
\check{f}\left(x_{1}, \ldots, x_{n}\right)=f_{A^{T}}\left(x_{1}, \ldots, x_{n}\right)=\sum_{i=1}^{n} x_{1}^{a_{i 1}} \cdots x_{n}^{a_{n i}}
$$

associated with the transpose matrix $A^{T}$. The list of bimodal singularities defined by invertible polynomials in three variables is given by Ebeling and Ploog [EP13, Table 2]. In order to relate Berglund-Hübsch transposition to mirror symmetry for K3 surfaces, we will deal only with the cases where the defining polynomial admits an extension to an invertible polynomial in four variables defining a K3 surface in a weighted projective space. Table 1.1] is obtained from [EP13, Table 4] by removing four cases not satisfying 


\begin{tabular}{ccccc}
\hline Name & $\left(\check{q}_{1}, \check{q}_{2}, \check{q}_{3}, \check{q}_{4} ; \check{h}\right)$ & $\check{F}(x, y, z, w)$ & $F(x, y, z, w)$ & $\left(q_{1}, q_{2}, q_{3}, q_{4} ; h\right)$ \\
\hline$J_{3,0}$ & $(2,6,9,1 ; 18)$ & $x^{6} y+y^{3}+z^{2}+w^{18}$ & $x^{6}+x y^{3}+z^{2}+w^{18}$ & $(3,5,9,1 ; 18)$ \\
$Z_{1,0}$ & $(2,4,7,1 ; 14)$ & $x^{5} y+x y^{3}+z^{2}+w^{14}$ & $x^{5} y+x y^{3}+z^{2}+w^{14}$ & $(2,4,7,1 ; 14)$ \\
$Q_{2,0}$ & $(2,4,5,1 ; 12)$ & $x^{4} y+y^{3}+x z^{2}+w^{12}$ & $x^{4} z+x y^{3}+z^{2}+w^{12}$ & $(3,7,12,2 ; 24)$ \\
$W_{1,0}$ & $(2,6,3,1 ; 12)$ & $x^{6}+y^{2}+y z^{2}+w^{12}$ & $x^{6}+y^{2} z+z^{2}+w^{12}$ & $(2,3,6,1 ; 12)$ \\
$S_{1,0}$ & $(2,4,3,1 ; 10)$ & $x^{5}+x y^{2}+y z^{2}+w^{10}$ & $x^{5} y+y^{2} z+z^{2}+w^{10}$ & $(3,5,10,2 ; 20)$ \\
$U_{1,0}$ & $(3,3,2,1 ; 9)$ & $x^{3}+x y^{2}+y z^{3}+w^{9}$ & $x^{3} y+y^{2} z+z^{3}+w^{9}$ & $(2,3,3,1 ; 9)$ \\
$E_{18}$ & $(1,3,4,1 ; 9)$ & $x^{5} z+y^{3}+z^{2} w+w^{9}$ & $x^{5}+y^{3}+x z^{2}+z w^{9}$ & $(3,5,6,1 ; 15)$ \\
$E_{19}$ & $(1,3,5,1 ; 10)$ & $x^{7} y+y^{3} w+z^{2}+w^{10}$ & $x^{7}+x y^{3}+z^{2}+y w^{10}$ & $(2,4,7,1 ; 14)$ \\
$E_{20}$ & $(1,4,6,1 ; 12)$ & $x^{11} w+y^{3}+z^{2}+w^{12}$ & $x^{11}+y^{3}+z^{2}+x w^{12}$ & $(6,22,33,5 ; 66)$ \\
$Z_{17}$ & $(1,2,3,1 ; 7)$ & $x^{4} z+x y^{3}+z^{2} w+w^{7}$ & $x^{4} y+y^{3}+x z^{2}+z w^{7}$ & $(2,4,5,1 ; 12)$ \\
$Z_{19}$ & $(1,3,5,1 ; 10)$ & $x^{9} w+x y^{3}+z^{2}+w^{10}$ & $x^{9} y+y^{3}+z^{2}+x w^{10}$ & $(4,18,27,5 ; 54)$ \\
$Q_{17}$ & $(1,2,3,1 ; 7)$ & $x^{5} y+y^{3} w+x z^{2}+w^{7}$ & $x^{5} z+x y^{3}+z^{2}+y w^{7}$ & $(1,3,5,1 ; 10)$ \\
$Q_{18}$ & $(1,3,4,1 ; 9)$ & $x^{8} w+y^{3}+x z^{2}+w^{9}$ & $x^{8} z+y^{3}+z^{2}+x w^{9}$ & $(3,16,24,5 ; 48)$ \\
$W_{18}$ & $(1,4,2,1 ; 8)$ & $x^{7} w+y^{2}+y z^{2}+w^{8}$ & $x^{7}+y^{2} z+z^{2}+x w^{8}$ & $(4,7,14,3 ; 28)$ \\
$S_{17}$ & $(1,3,2,1 ; 7)$ & $x^{6} w+x y^{2}+y z^{2}+w^{7}$ & $x^{6} y+y^{2} z+z^{2}+x w^{7}$ & $(1,2,4,1 ; 8)$ \\
$U_{16}$ & $(1,2,2,1 ; 6)$ & $x^{5} w+y^{2} z+y z^{2}+w^{6}$ & $x^{5}+y^{2} z+y z^{2}+x w^{6}$ & $(3,5,5,2 ; 15)$ \\
\hline
\end{tabular}

Table 1.1: Transposition of invertible polynomials

this condition. Here, the invertible polynomial $F(x, y, z, w)$ is a four-variable extension of an invertible polynomial $f(x, y, z)$ in three variables, whose Berglund-Hübsch transpose $\check{f}(x, y, z)$ defines a bimodal singularity. Note that the Berglund-Hübsch transpose $\breve{F}(x, y, z, w)$ of $F(x, y, z, w)$ may not be a four-variable extension of $\breve{f}(x, y, z)$ in general;

$$
\check{F}(x, y, z, 0) \neq \check{f}(x, y, z) .
$$

The weight $\left(q_{1}, q_{2}, q_{3}, q_{4}\right)$ is the primitive weight which makes $F$ into a weighted homogeneous polynomial of degree $h$. The weight $\left(\check{q}_{1}, \check{q}_{2}, \check{q}_{3}, \check{q}_{4}\right)$ is defined similarly as the primitive weight which makes $\check{F}$ weighted homogeneous of degree $\check{h}$.

The group of maximal diagonal symmetries of $F$ is defined by

$$
G_{\max }(F):=\left\{\operatorname{diag}(\alpha, \beta, \gamma, \delta) \in G L_{4}(\mathbb{C}) \mid F(\alpha x, \beta y, \gamma z, \delta w)=F(x, y, z, w)\right\} .
$$

Since any element of $G_{\max }(F)$ is contained in the subgroup $(\mathbb{Q} / \mathbb{Z})^{4} \subset\left(\mathbb{C}^{\times}\right)^{4}$, we can use an additive notation and write $\frac{1}{n}(a, b, c, d)$ for $\left(\zeta_{n}^{a}, \zeta_{n}^{b}, \zeta_{n}^{c}, \zeta_{n}^{d}\right)$, where $\zeta_{n}=\exp (2 \pi \sqrt{-1} / n)$. In the additive notation, the group $G_{\max }(F)$ of maximal diagonal symmetries is generated by the column vectors

$$
\rho_{i}=\left(\left(A^{-1}\right)_{1 i},\left(A^{-1}\right)_{2 i},\left(A^{-1}\right)_{3 i},\left(A^{-1}\right)_{4 i}\right), \quad i=1,2,3,4,
$$

of the inverse matrix of $A$.

For a subgroup $G$ of $G_{\max }(F)$ containing $J=J(F)=\frac{1}{h}\left(q_{1}, q_{2}, q_{3}, q_{4}\right)$, the natural action of $G$ on $\mathbb{C}^{4}$ induces an action of $\bar{G}=G /\langle J\rangle$ on the weighted projective space $\mathbb{P}=\mathbb{P}\left(q_{1}, q_{2}, q_{3}, q_{4}\right)$. This gives an action of $\bar{G}$ on the hypersurface

$$
Y:=\{[x: y: z: w] \in \mathbb{P} \mid F(x, y, z, w)=0\},
$$


since $F(x, y, z, w)$ is invariant under the action of $\bar{G}$. The quotient stack $\mathbb{X}=[\mathbb{P} / \bar{G}]$ is a toric stack, and we write the group of characters of its dense torus as $M$. Since $F$ has an isolated critical point at the origin, the hypersurface $\mathbb{Y}$ of $\mathbb{X}$ defined by $F$ is a smooth Deligne-Mumford stack. We write the Newton polytope of $F$ in $M_{\mathbb{R}}=M \otimes_{\mathbb{Z}} \mathbb{R}$ as $\Delta_{(F, G)}$.

The Berglund-Hübsch transpose of $G$ is defined by

$\check{G}:=\left\{\check{\rho}_{1}^{r_{1}} \check{\rho}_{2}^{r_{2}} \check{\rho}_{3}^{r_{3}} \check{\rho}_{4}^{r_{4}} \in G_{\max }(\check{F}) \mid \begin{array}{llll}r_{1} & r_{2} & r_{3} & r_{4}\end{array}\right) A^{-1}\left(\begin{array}{c}a_{1} \\ a_{2} \\ a_{3} \\ a_{4}\end{array}\right) \in \mathbb{Z}$ for all $\left.\rho_{1}^{a_{1}} \rho_{2}^{a_{2}} \rho_{3}^{a_{3}} \rho_{4}^{a_{4}} \in G\right\}$.

The Newton polytope $\Delta_{(\check{F}, \breve{G})}$ of $\check{F}$ in $\check{M}_{\mathbb{R}}$ is defined similarly, where $\check{M}$ is the group of characters of the dense torus in the toric stack $\check{\mathbb{X}}=\left[\mathbb{P}\left(\check{q}_{1}, \check{q}_{2}, \check{q}_{3}, \check{q}_{4}\right) / \check{G}\right]$.

Let $N=\operatorname{Hom}(M, \mathbb{Z})$ be the dual lattice of $M$. The polar dual of a polytope $\Delta$ in $M_{\mathbb{R}}$ is defined by

$$
\Delta^{\circ}=\left\{n \in N_{\mathbb{R}} \mid\langle n, m\rangle \geq-1 \text { for any } m \in \Delta\right\} .
$$

A lattice polytope is reflexive if the polar dual polytope is a lattice polytope. Polar duality of reflexive polytope is an essential ingredient of mirror construction by Batyrev [Bat94]. In this paper, we give a relation between transposition and polar duality for bimodal singularities.

Theorem 1.1. For any polynomial $F$ in Table 1.1, there is an isomorphism $\varphi: N \stackrel{\sim}{\rightarrow} \check{M}$ of abelian groups and a reflexive polytope $\Delta \subset M_{\mathbb{R}}$ such that

$$
\Delta_{(F, G)} \subset \Delta \quad \text { and } \quad \Delta_{(\check{F}, \check{G})} \subset \varphi\left(\Delta^{\circ}\right),
$$

where $G$ is the unique lift of $G_{\max }(f)$ to a subgroup of $G_{\max }(F) \cap S L_{4}(\mathbb{C})$.

This shows that for K3 surfaces associated with bimodal singularities, BerglundHübsch mirror construction can be regarded as a special case of Batyrev mirror construction. This generalizes a related result for unimodal singularities by Kobayashi Kob08, Thereom 4.3.9].

The group $\bar{G}$, which is trivial for unimodal singularities, comes from homological mirror symemtry [Kon95] for singularities.

Conjecture 1.2. For any invertible polynomial $f$, there is an equivalence

$$
D^{b} \mathfrak{F} \mathfrak{u k} \check{f} \cong D_{\text {sing }}^{b}\left(\mathrm{gr}^{L_{\max }} R\right)
$$

of triangulated categories.

The category $\mathfrak{F} \mathfrak{u k} \check{f}$ on the left hand side is the Fukaya-Seidel category, whose objects are vanishing cycles of $\check{f}$ and whose spaces of morphisms are Lagrangian intersection Floer complexes [Sei08]. It is a categorification of the Milnor lattice, which is an important invariant in singularity theory. The category $D_{\text {sing }}^{b}\left(\operatorname{gr}^{L_{\max }} R\right)$ on the right hand side is the stable derived category, also known as the singularity category, defined as the quotient

$$
D_{\text {sing }}^{b}\left(\operatorname{gr}^{L_{\max }} R\right)=D^{b}\left(\operatorname{gr}^{L_{\max }} R\right) / D^{\text {perf }}\left(\operatorname{gr}^{L_{\max }} R\right)
$$


of the bounded derived category $D^{b}\left(\operatorname{gr}^{L_{\max }} R\right)$ of finitely-generated $L_{\max }$-graded $R$-modules by the full subcategory $D^{\text {perf }}\left(\operatorname{gr}^{L_{\max }} R\right)$ consisting of bounded complexes of projectives. The ring

$$
R=\mathbb{C}\left[x_{1}, \ldots, x_{n}\right] /\left(f\left(x_{1}, \ldots, x_{n}\right)\right)
$$

is the coordinate ring of zero of $f$, which is graded by the abelian group

$$
L_{\max }=\mathbb{Z} \vec{x}_{1} \oplus \cdots \oplus \mathbb{Z} \vec{x}_{n} \oplus \mathbb{Z} \vec{c} /\left(a_{i 1} \vec{x}_{1}+\cdots+a_{i n} \vec{x}_{n}-\vec{c}\right)_{i=1}^{n}
$$

of rank one. Stable derived categories are first introduced by Buchweitz [Buc87] motivated by a work of Eisenbud [Eis80, and later by Orlov Orl04 following an idea of Kontsevich.

In the case of bimodal singularities, Ebeling and Ploog gave the following geometric construction of Coxeter-Dynkin diagrams:

Theorem 1.3 ([EP13, Theorem 7]). For any bimodal singularity $\check{f}$ in [EP13, Table 2], there is a distinguished basis $\left(\Delta_{i}\right)_{i=1}^{\mu}$ of vanishing cycles and a collection $\left(\mathcal{E}_{i}\right)_{i=1}^{\mu}$ of objects of $D^{b} \operatorname{coh} \mathbb{Y}$ such that

$$
\Delta_{i} \cdot \Delta_{j}=-\sum_{k}(-1)^{k} \operatorname{dim} \operatorname{Ext}^{k}\left(\mathcal{E}_{i}, \mathcal{E}_{j}\right)
$$

Here $\Delta_{i} \cdot \Delta_{j}$ is the intersection number of vanishing cycles. Theorem 1.4 below shows that Theorem 1.3 is an evidence to Conjecture 1.2 .

\section{Theorem 1.4. Conjecture 1.2 implies Theorem 1.3 .}

Conjecture 1.2 is known for Sebastiani-Thom sum of polynomials of types A and D [FU11, FU13]. Since Fukaya-Seidel categories are invariant under suspension [Sei10], Conjecture 1.2 for a suspension of a polynomial in two variables is reduced to the case of curve singularities, which is announced by Takahashi [Tak09. Together, they cover 10 out of 20 cases in [EP13, Table 2], and Theorem 1.4 gives an alternative proof of Theorem 1.3 for such cases.

Acknowledgment: K. U. is supported by JSPS Grant-in-Aid for Young Scientists No. 24740043. A part of this work is done while K. U. is visiting Korea Institute for Advanced Study, whose hospitality and wonderful working environment is gratefully acknowledged.

\section{Transposition and polar duality}

Let $F=\sum_{i=1}^{n} x_{1}^{a_{i 1}} \cdots x_{n}^{a_{i n}}$ be an invertible polynomial associated with a matrix $A=$ $\left(a_{i j}\right)_{i, j=1}^{n}$. The group $L_{\max }$ defined in (1.10) is the group of characters of

$$
K_{\max }=\left\{\left(\alpha_{1}, \ldots, \alpha_{n}\right) \in\left(\mathbb{C}^{\times}\right)^{n} \mid \alpha_{1}^{a_{11}} \cdots \alpha_{n}^{a_{1 n}}=\cdots=\alpha_{1}^{a_{n 1}} \cdots \alpha_{n}^{a_{n n}}\right\} .
$$

The group

$$
G_{\max }(F)=\left\{\operatorname{diag}\left(\alpha_{1}, \ldots, \alpha_{n}\right) \in G L_{n}(\mathbb{C}) \mid \prod_{j=1}^{n} \alpha_{j}^{a_{i j}}=1 \text { for any } i=1, \ldots, n\right\}
$$


of maximal diagonal symmetries of $F$ is the kernel of the map

$$
\begin{array}{ccc}
K_{\max } & \rightarrow & \mathbb{C}^{\times} \\
\Psi & & \Psi \\
\left(\alpha_{1}, \ldots, \alpha_{n}\right) & \mapsto & \alpha_{1}^{a_{11}} \cdots \alpha_{n}^{a_{1 n}},
\end{array}
$$

so that one has an exact sequence

$$
1 \rightarrow G_{\max }(F) \rightarrow K_{\max } \rightarrow \mathbb{C}^{\times} \rightarrow 1 .
$$

Let $\left(q_{1}, \ldots, q_{n}\right)$ be the primitive weight which makes $F$ weighted homogeneous of degree $h$. Set $J(F)=\operatorname{diag}\left(\zeta_{h}^{q_{1}}, \ldots, \zeta_{h}^{q_{n}}\right) \in G_{\max }(F)$ where $\zeta_{h}=\exp (2 \pi \sqrt{-1} / h)$ is the primitive $h$-th root of unity. Then one has an exact sequence

$$
1 \rightarrow \mathbb{C}^{\times} \stackrel{\varphi}{\rightarrow} K_{\max } \rightarrow \bar{G}_{\max }(F) \rightarrow 1
$$

where $\varphi(\alpha)=\left(\alpha^{q_{1}}, \ldots, \alpha^{q_{n}}\right)$ and $\bar{G}_{\max }(F)=G_{\max }(F) /\langle J(F)\rangle$.

Let $G$ be a subgroup of $G_{\max }(F)$ containing $J(F)$ and set $\bar{G}=G /\langle J(F)\rangle$. The inverse image of $\bar{G}$ in (2.5) will be denoted by $K$ :

$$
1 \rightarrow \mathbb{C}^{\times} \stackrel{\varphi}{\rightarrow} K \rightarrow \bar{G} \rightarrow 1 .
$$

The group of characters of $K$ will be denoted by $L$. Let $\mathbb{T}$ be the cokernel of the inclusion $K \subset\left(\mathbb{C}^{\times}\right)^{n}$, which is isomorphic to $\left(\mathbb{C}^{\times}\right)^{n-1}$. Let $M=\operatorname{Hom}\left(\mathbb{T}, \mathbb{C}^{\times}\right)$be the group of characters of $\mathbb{T}$. One has an exact sequence

$$
1 \rightarrow K \rightarrow\left(\mathbb{C}^{\times}\right)^{n} \rightarrow \mathbb{T} \rightarrow 1
$$

of abelian groups, which induces an exact sequence

$$
1 \rightarrow M \rightarrow \mathbb{Z}^{n} \rightarrow L \rightarrow 1
$$

of groups of characters. The natural action of $\left(\mathbb{C}^{\times}\right)^{n}$ on $\mathbb{C}^{n}=\operatorname{Spec} U$ restricts to an action of $K$, which induces an $L$-grading on the coordinate ring $U=\mathbb{C}\left[x_{1}, \ldots, x_{n}\right]$. The quotient stack $\mathbb{X}=\left[\left(\mathbb{C}^{n} \backslash \mathbf{0}\right) / K\right]$ is a toric Deligne-Mumford stack with the dense torus $\mathbb{T}$ and the Picard group $L$.

The polynomial $F$ gives a section of a line bundle on $\mathbb{X}$, so that its Newton polytope

$$
\Delta_{F}=\operatorname{Conv}\left\{\left(a_{i 1}, \ldots, a_{i n}\right)\right\}_{i=1}^{n} \subset \mathbb{R}^{n}
$$

is a lattice polytope in the translate of $M_{\mathbb{R}}=M \otimes \mathbb{R}$. This polytope, considered as a lattice polytope in $M_{\mathbb{R}}$, will be denoted by $\Delta_{(F, G)}$. It is well-defined up to translation by a lattice vector. Let $S=U /(F)$ be the quotient ring of $U$ by the ideal generated by the polynomial $F$. Since $F$ has an isolated critical point at the origin, the hypersurface $\mathbb{Y}=[(\operatorname{Spec} S \backslash \mathbf{0}) / K] \subset \mathbb{X}$ is a smooth Deligne-Mumford stack.

The Berglund-Hübsch transpose of the pair $(F, G)$ is defined in [BH93, Kra] as the pair $(\check{F}, \check{G})$ of the invertible polynomial $\check{F}=\sum_{i=1}^{n} x_{1}^{a_{1 i}} \cdots x_{n}^{a_{n i}}$ and the group

$$
\check{G}:=\left\{\check{\rho}_{1}^{r_{1}} \cdots \check{\rho}_{n}^{r_{n}} \in G_{\max }(\check{F}) \mid\left(\begin{array}{lll}
r_{1} & \cdots & r_{n}
\end{array}\right) A^{-1}\left(\begin{array}{c}
a_{1} \\
\vdots \\
a_{n}
\end{array}\right) \in \mathbb{Z} \text { for all } \rho_{1}^{a_{1}} \cdots \rho_{n}^{a_{n}} \in G\right\} .
$$

One can easily see that 
- the group $\check{G}$ contains $J(\check{F})$ if and only if the group $G$ is contained in $S L_{n}(\mathbb{C})$, and

- the group $\check{G}$ is contained in $S L_{n}(\mathbb{C})$ if and only if the group $G$ contains $J(F)$.

Assume that $G$ (and hence $\check{G}$ ) contains $J(F)$ and is contained in $S L_{n}(\mathbb{C})$. The toric stack $\check{\mathbb{X}}$, its hypersurface $\check{\mathbb{Y}}$, the dense torus $\check{\mathbb{T}} \subset \check{\mathbb{X}}$, the lattice $\check{M}=\operatorname{Hom}\left(\check{\mathbb{X}}, \mathbb{C}^{\times}\right)$and the lattice polytope $\Delta_{(\check{F}, \check{G})} \subset \check{M}_{\mathbb{R}}$ are defined in the same way as $(F, G)$.

For a lattice polytope $\Delta$ in $M_{\mathbb{R}}$, its polar dual polytope $\Delta^{\circ}$ is defined by

$$
\Delta^{\circ}=\left\{n \in N_{\mathbb{R}} \mid\langle n, m\rangle \geq-1 \text { for any } m \in \Delta\right\},
$$

where $N=\operatorname{Hom}(M, \mathbb{Z})$ and $N_{\mathbb{R}}=N \otimes_{\mathbb{Z}} \mathbb{R}$. A lattice polytope is reflexive if its polar dual polytope is a lattice polytope. According to Batyrev [Bat94], the mirror of an anticanonical hypersurface of a toric weak Fano manifold associated with a reflexive polytope is an anti-canonical hypersurface of a toric weak Fano manifold associated with the polar dual polytope. The following problem asks the relation between transposition and polar duality:

Problem 2.1. There exist a lattice isomorphism $\varphi: N \stackrel{\sim}{\rightarrow} \check{M}$ and a reflexive polytope $\Delta \subset M$ such that $\Delta_{(F, G)} \subset \Delta$ and $\Delta_{(\breve{F}, \breve{G})} \subset \varphi\left(\Delta^{\circ}\right)$.

Theorem 1.1 gives an affirmative answer to Problem 2.1 for invertible polynomials in Table 1.1 with a specific group $G$ coming from mirror symmetry for singularities.

\section{Homological mirror symmetry for singularities}

Let $f=\sum_{i=1}^{n} x_{1}^{a_{i 1}} \cdots x_{n}^{a_{i n}}$ be an invertible polynomial and $\check{f}$ be its Berglund-Hübsch transpose. The coordinate ring $R=\mathbb{C}\left[x_{1}, \ldots, x_{n}\right] /(f)$ is graded by the abelian group $L=L_{\max }(f)$ of rank one defined in (1.10). By perturbing $\check{f}$ slightly, one obtains an exact symplectic Lefschetz fibration [Sei10, Section 6], which we write as $\check{f}$ again by abuse of notation. Choose a distinguished basis $\left(\Delta_{i}\right)_{i=1}^{\mu}$ of vanishing cycles, where $\mu$ is the Milnor number of $\check{f}$. The Fukaya category of Lefschetz fibration, also known as the Fukaya-Seidel category, is an $A_{\infty}$-category such that

- the set of objects is $\left(\Delta_{i}\right)_{i=1}^{\mu}$, and

- the space of morphisms are given by

$$
\operatorname{hom}^{*}\left(\Delta_{i}, \Delta_{j}\right)= \begin{cases}0 & i>j \\ \mathbb{C} \cdot \operatorname{id}_{\Delta_{i}} & i=j \\ \operatorname{CF}^{*}\left(\Delta_{i}, \Delta_{j}\right) & i<j\end{cases}
$$

where $\mathrm{CF}^{*}\left(\Delta_{i}, \Delta_{j}\right)$ is the Lagrangian intersection Floer complex, and

- the composition is defined by virtual couting of holomorphic disks with Lagrangian boundary conditions. 
We refer the reader to [Sei08] for the definition of Fukaya-Seidel categories. The quasiequivalence class of the derived Fukaya-Seidel category $D^{b} \mathfrak{F} \mathfrak{k} \mathfrak{f}$ is independ of various auxiliary choices in the definition of $D^{b} \mathfrak{F} \mathfrak{u k} \check{f}$, such as the choices of a perturbation or a distinguished basis of vanishing cycles. The Grothendieck group $K(\mathfrak{F u k} \check{f})$ of the FukayaSeidel category is freely generated by the distinguished basis of vanishing cycles. The Euler number of the Floer complex is equal to minus the intersection number of Lagrangian submanifolds;

$$
\sum_{k}(-1)^{k} \operatorname{dim} \mathrm{CF}^{k}\left(\Delta_{i}, \Delta_{j}\right)=-\Delta_{i} \cdot \Delta_{j}
$$

The lattice of vanishing cycles equipped with the intersection form is called the Milnor lattice. The Euler form on the Grothendieck group of a triangulated category is defined by

$$
\chi(\mathcal{E}, \mathcal{F})=\sum_{k}(-1)^{k} \operatorname{dim} \operatorname{Hom}^{k}(\mathcal{E}, \mathcal{F})
$$

The Fukaya-Seidel category is a categorification of the Milnor lattice, in the sense that the Grothendieck group equipped with the symmetrized Euler form

$$
\left(\Delta_{i}, \Delta_{j}\right)=\chi\left(\Delta_{i}, \Delta_{j}\right)+(-1)^{n-1} \chi\left(\Delta_{j}, \Delta_{i}\right)
$$

is isomorphic to the Milnor lattice up to sign;

$$
\left(\Delta_{i}, \Delta_{j}\right)=-\Delta_{i} \cdot \Delta_{j}
$$

Under Conjecture 1.2, the graded stable derived category $D_{\text {sing }}^{b}\left(\operatorname{gr}^{L} R\right)$ is also a categorification of the Milnor lattice of $\check{f}$, where $R$ is the coordinate ring $\mathbb{C}\left[x_{1}, \ldots, x_{n}\right] /(f)$ of the singularity defined by the transpose of $\check{f}$, and $L=L_{\max }(f)$ is the maximal grading group for $f$.

Let $F\left(x_{1}, \ldots, x_{n+1}\right) \in \mathbb{C}\left[x_{1}, \ldots, x_{n+1}\right]$ be a polynomial in $n+1$ variables satisfying the following assumption:

\section{Assumption 3.1.}

- $F\left(x_{1}, \ldots, x_{n}, 0\right)=f\left(x_{1}, \ldots, x_{n}\right)$.

- $F$ is weighted homogeneous of degree $\left(q_{1}, \ldots, q_{n+1} ; h\right)$ satisfying $q_{1}+\cdots+q_{n+1}=h$.

- F has an isolated critical point at the origin.

- For any $g=\operatorname{diag}\left(\alpha_{1}, \ldots, \alpha_{n}\right) \in G_{\max }(f)$, the polynomial $F$ is invariant under the action of its lift $\widetilde{g}=\operatorname{diag}\left(\alpha_{1}, \ldots, \alpha_{n}, \alpha_{1}^{-1} \cdots \alpha_{n}^{-1}\right) \in S L_{n+1}(\mathbb{C})$.

This condition is satisfied for all polynomials in [EP13, Table 2]. We write the image of the injective map $G_{\max }(f) \ni g \mapsto \widetilde{g} \in G_{\max }(F) \cap S L_{n+1}(\mathbb{C})$ as $G$. The group $G$ defines the group $K$ fitting in the exact sequence (2.6). Let $S=\mathbb{C}\left[x_{1}, \ldots, x_{n}\right] /(F)$ be the coordinate ring of the zero of $F$, which is graded by the group $L$ of characters of $K$. The corresponding projective stack will be denoted by $\mathbb{Y}=\operatorname{Proj} S=[(\operatorname{Spec} S \backslash \mathbf{0}) / K]$.

We have the following two theorems: 
Theorem 3.2 ([0r109, Theorem 2.13]). There is an equivalence

$$
\Psi: D_{\text {sing }}^{b}\left(\mathrm{gr}^{L} S\right) \rightarrow D^{b} \operatorname{coh} \mathbb{Y}
$$

of triangulated categories.

Theorem 3.3 ([Ued, Theorem 1.1]). There is a functor

$$
\Phi: D_{\text {sing }}^{b}\left(\operatorname{gr}^{L} R\right) \rightarrow D_{\text {sing }}^{b}\left(\operatorname{gr}^{L} S\right)
$$

such that

$$
\operatorname{Hom}^{i}(\Phi(\mathcal{E}), \Phi(\mathcal{F})) \cong \operatorname{Hom}^{i}(\mathcal{E}, \mathcal{F}) \oplus \operatorname{Hom}^{n-i-1}(\mathcal{F}, \mathcal{E})
$$

Although both theorems are stated for $\mathbb{Z}$-graded rings in the references, the generalization to the $L$-graded case is straighforward. Theorem 3.4 below contains Theorem 1.4 as a special case:

Theorem 3.4. Let $f$ be an invertible polynomial in $n$ variables satisfying Conjecture 1.2, and $F$ be a polynomial in $n+1$ variables satisfying Assupmtion 3.1. Then for any distinguished basis $\left(\Delta_{i}\right)_{i=1}^{\mu}$ of vanishing cycles of $\tilde{f}$, there is a collection $\left(\mathcal{E}_{i}\right)_{i=1}^{\mu}$ of objects of $D^{b} \operatorname{coh} \mathbb{Y}$ satisfying

$$
\Delta_{i} \cdot \Delta_{j}=-\chi\left(\mathcal{E}_{i}, \mathcal{E}_{j}\right)
$$

Proof. The distinguished basis $\left(\Delta_{i}\right)_{i=1}^{\mu}$ of vanishing cycles are objects of the Fukaya-Seidel category. Let $\left(\mathcal{F}_{i}\right)_{i=1}^{\mu}$ be their images in $D_{\text {sing }}^{b}\left(\operatorname{gr}^{L} R\right)$ under the equivalence (1.7), so that

$$
\chi\left(\Delta_{i}, \Delta_{j}\right)=\chi\left(\mathcal{F}_{i}, \mathcal{F}_{j}\right) .
$$

If we set $\mathcal{G}_{i}=\Phi\left(\mathcal{F}_{i}\right)$ and $\mathcal{E}_{i}=\Psi\left(\mathcal{G}_{i}\right)$ for $i=1, \ldots, \mu$, then we have

$$
\chi\left(\mathcal{G}_{i}, \mathcal{G}_{j}\right)=\chi\left(\mathcal{F}_{i}, \mathcal{F}_{j}\right)+(-1)^{n-1} \chi\left(\mathcal{F}_{j}, \mathcal{F}_{i}\right)
$$

by (3.8) and

$$
\chi\left(\mathcal{E}_{i}, \mathcal{E}_{j}\right)=\chi\left(\mathcal{G}_{i}, \mathcal{G}_{j}\right)
$$

by Theorem 3.2. Now (3.9) follows from (3.4), (3.5), (3.11) and (3.12).

\section{Proof of Theorem 1.1}

\section{$4.1 \quad J_{3,0}$-singularity}

The invertible polynomial

$$
\check{f}=x^{6} y+y^{3}+z^{2}
$$

defines a $J_{3,0}$-singularity. Its Berglund-Hübsch transpose

$$
f=x^{6}+x y^{3}+z^{2}
$$


is weighted homogeneous of degree $\left(q_{1}, q_{2}, q_{3} ; h\right)=(3,5,9 ; 18)$. The group of maximal diagonal symmetries is given by

$$
G_{\max }(f)=\left\{(\alpha, \beta, \gamma) \in\left(\mathbb{C}^{\times}\right)^{3} \mid \alpha^{6}=\alpha \beta^{3}=\gamma^{2}=1\right\}=\left\langle J(f), \frac{1}{2}(0,0,1)\right\rangle
$$

where $J(f)=\frac{1}{18}(3,5,9)$. The invertible polynomial

$$
F=x^{6}+x y^{3}+z^{2}+w^{18}
$$

is a four-variable extension of $\check{f}$ satisfying Assupmtion 3.1. The subgroup $G$ of $G_{\max }(F)$ is given by

$$
G=\left\langle J(F), \frac{1}{2}(0,0,1,1)\right\rangle
$$

where $J(F)=\frac{1}{18}(3,5,9,1)$. The lattice $M$ is given by

$$
M=\left\{(i, j, k, l) \in \mathbb{Z}^{4} \mid 3 i+5 j+9 k+l=0 \text { and } k+l \equiv 0 \bmod 2\right\} .
$$

For each monomial $x^{a_{i 1}} y^{a_{i 2}} z^{a_{i 3}} w^{a_{i 4}}$ in $F$, the Laurent monomial $x^{a_{i 1}} y^{a_{i 2}} z^{a_{i 3}} w^{a_{i 4}} / x y z w$ defines a regular function on $\mathbb{T}$, and hence gives a lattice point $\left(a_{i 1}-1, a_{i 2}-1, a_{i 3}-1, a_{i 4}-1\right)$ in $M$. By identifying $M$ with $\mathbb{Z}^{3}$ by the basis

$$
e_{1}=(5,-1,-1,-1), \quad e_{2}=(0,2,-1,-1), \quad e_{3}=(-1,-1,1,-1),
$$

these points are given by

\begin{tabular}{c|c|c|c}
$x^{6}$ & $x y^{3}$ & $z^{2}$ & $w^{18}$ \\
\hline$(1,0,0)$ & $(0,1,0)$ & $(0,0,1)$ & $(-2,-6,-9)$
\end{tabular}.

The Newton polytope $\Delta_{(F, G)}$ is the convex hull of these points. Similarly, we choose the basis of the group

$$
\check{M}:=\left\{(i, j, k, l) \in \mathbb{Z}^{4} \mid 2 i+6 j+9 k+l=0\right\}
$$

of characters of the quotient of the dense torus of $\check{\mathbb{X}}=\mathbb{P}(2,6,9,1)$ as

$$
\check{e}_{1}=(1,0,0,-2), \quad \check{e}_{2}=(0,1,0,-6), \quad \check{e}_{3}=(0,0,1,-9),
$$

so that the Newton polytope $\Delta_{(\check{F}, \breve{G})}$ of $\check{F} / x y z w$ is the convex hull of

$$
\begin{array}{c|c|c|c}
x^{6} y & y^{3} & z^{2} & w^{18} \\
\hline(5,0,-1) & (-1,2,-1) & (-1,-1,1) & (-1,-1,-1)
\end{array} .
$$

Instead of $\Delta_{(F, G)}$ which is not reflexive, take the polytope

$$
\Delta:=\{(1,0,0),(0,1,0),(0,0,1),(-2,-6,-9),(-1,-2,-4)\},
$$

which clearly contains $\Delta_{(F, G)}$. The polytope $\Delta$ is reflexive, whose polar dual polytope is given by

$$
\Delta^{\circ}:=\{(5,0,-1),(-1,2,-1),(-1,-1,1),(-1,-1,-1),(7,-1,-1)\} .
$$

$\Delta^{\circ}$ clearly contains $\Delta_{(\check{F}, \breve{G})}$, so that Theorem 1.1 holds for this case. 


\section{$4.2 \quad Z_{1,0}$-singularity}

The invertible polynomial $f=x^{5} y+x y^{3}+z^{2}$ is weighted homogeneous of degree $\left(q_{1}, q_{2}, q_{3} ; h\right)=$ $(2,4,7 ; 14)$. The group of maximal diagonal symmetries is given by

$$
G_{\max }(f)=\left\langle J(f), \frac{1}{2}(1,1,0)\right\rangle .
$$

The four-variable extension $F=x^{5} y+x y^{3}+z^{2}+w^{14}$ of $f$ is weighted homogeneous of weight $(2,4,7,1)$ and satisfies Assupmtion 3.1. The subgroup $G$ of $G_{\max }(F)$ is given by $G=\left\langle J(F), \frac{1}{2}(1,1,0,0)\right\rangle$. The lattice $M$ is given by

$$
M=\left\{(i, j, k, l) \in \mathbb{Z}^{4} \mid 2 i+4 j+7 k+l=0 \text { and } i+j \equiv 0 \bmod 2\right\} .
$$

We choose a basis of $M$ as

$$
e_{1}=(1,1,0,-6), \quad e_{2}=(1,-1,0,2), \quad e_{3}=(0,0,1,-7) .
$$

With respect this basis, the polytope $\Delta_{(F, G)}$ is the convex hull of

$$
\begin{array}{c|c|c|c}
x^{5} y & x y^{3} & z^{2} & w^{14} \\
\hline(2,2,-1) & (1,-1,-1) & (-1,0,1) & (-1,0,-1)
\end{array} .
$$

Similarly, we choose the basis of the group

$$
\check{M}:=\left\{(i, j, k, l) \in \mathbb{Z}^{4} \mid 2 i+4 j+7 k+l=0\right\}
$$

as

$$
\check{e}_{1}=(2,1,-1,-1), \quad \check{e}_{2}=(2,-1,0,0), \quad \check{e}_{3}=(-1,-1,1,-1),
$$

so that the Newton polytope $\Delta_{(\breve{F}, \breve{G})}$ of $\check{F} / x y z w$ is the convex hull of

$$
\begin{array}{c|c|c|c}
x^{5} y & x y^{3} & z^{2} & w^{14} \\
\hline(1,1,0) & (1,-1,0) & (0,0,1) & (-6,2,-7)
\end{array} .
$$

The polytope

$$
\Delta:=\{(2,2,-1),(1,-1,-1),(-1,0,1),(-1,0,-1),(2,3,-1),(0,-1,-1)\}
$$

contains $\Delta_{(F, G)}$, and its polar dual polytope

$$
\Delta^{\circ}:=\{(1,1,0),(1,-1,0),(0,0,1),(-6,2,-7),(0,2,-1),(-2,0,-3)\}
$$

contains $\Delta_{(\check{F}, \check{G})}$. 


\section{$4.3 \quad Q_{2,0}$-singularity}

The invertible polynomial $f=x^{4} z+x y^{3}+z^{2}$ is weighted homogeneous of degree $\left(q_{1}, q_{2}, q_{3} ; h\right)=$ $(3,7,12 ; 24)$. The group of maximal diagonal symmetries is given by

$$
G_{\max }(f)=\langle J(f)\rangle \text {. }
$$

The four-variable extension $F=x^{4} z+x y^{3}+z^{2}+w^{12}$ of $f$ is weighted homogeneous of weight $(3,7,12,2)$ and satisfies Assupmtion 3.1. The subgroup $G$ of $G_{\max }(F)$ is given by $G=\langle J(F)\rangle$. The lattice $M$ is given by

$$
M=\left\{(i, j, k, l) \in \mathbb{Z}^{4} \mid 3 i+7 j+12 k+2 l=0\right\} .
$$

We choose a basis of $M$ as

$$
e_{1}=(0,0,-1,6), \quad e_{2}=(2,0,-1,3), \quad e_{3}=(1,-1,0,2) .
$$

With respect this basis, the polytope $\Delta_{(F, G)}$ is the convex hull of

$$
\begin{array}{c|c|c|c}
z^{2} & w^{12} & x y^{3} & x^{4} z \\
\hline(0,-1,1) & (2,-1,1) & (0,1,-2) & (-1,1,1)
\end{array} .
$$

Similarly, we choose the basis of the group

$$
\check{M}:=\left\{(i, j, k, l) \in \mathbb{Z}^{4} \mid 2 i+4 j+5 k+l=0\right\}
$$

as

$$
\check{e}_{1}=(-1,0,0,2), \quad \check{e}_{2}=(1,1,-1,-1), \quad \check{e}_{3}=(1,-2,1,1) \text {, }
$$

so that the Newton polytope $\Delta_{(\breve{F}, \breve{G})}$ of $\check{F} / x y z w$ is the convex hull of

\begin{tabular}{c|c|c|c}
$x z^{2}$ & $w^{7} z$ & $x^{4} y$ & $y^{3}$ \\
\hline$(-1,-1,0)$ & $(3,1,1)$ & $(0,2,1)$ & $(0,0,-1)$
\end{tabular}.

The polytope

$$
\Delta:=\{(0,1,-2),(0,1,1),(-1,1,1),(0,-1,1),(2,-1,1)\}
$$

contains $\Delta_{(F, G)}$, and its polar dual polytope

$$
\Delta^{\circ}:=\{(-1,-1,0),(0,-1,0),(0,0,-1),(6,3,2),(0,3,2)\}
$$

contains $\Delta_{(\check{F}, \check{G})}$.

\section{$4.4 W_{1,0}$-singularity}

The invertible polynomial $f=x^{6}+y^{2} z+z^{2}$ is weighted homogeneous of degree $\left(q_{1}, q_{2}, q_{3} ; h\right)=$ $(2,3,6 ; 12)$. The group of maximal diagonal symmetries is given by

$$
G_{\max }(f)=\left\langle J(f), \frac{1}{2}(1,0,0)\right\rangle \text {. }
$$


The four-variable extension $F=x^{6}+y^{2} z+z^{2}+w^{12}$ of $f$ is weighted homogeneous of weight $(2,3,6,1)$ and satisfies Assupmtion 3.1. The subgroup $G$ of $G_{\max }(F)$ is given by $G=\left\langle J(F), \frac{1}{2}(1,0,0,1)\right\rangle$. The lattice $M$ is given by

$$
M=\left\{(i, j, k, l) \in \mathbb{Z}^{4} \mid 2 i+3 j+6 k+l=0 \text { and } i+l \equiv 0 \bmod 2\right\} .
$$

We choose a basis of $M$ as

$$
e_{1}=(1,1,0,-5), \quad e_{2}=(1,-1,0,1), \quad e_{3}=(0,0,1,-6) .
$$

With respect this basis, the polytope $\Delta_{(F, G)}$ is the convex hull of

$$
\begin{array}{c|c|c|c}
z^{2} & w^{12} & x^{6} & y^{2} z \\
\hline(-1,0,1) & (-1,0,-1) & (2,3,-1) & (0,-1,0)
\end{array}
$$

Similarly, we choose the basis of the group

$$
\check{M}:=\left\{(i, j, k, l) \in \mathbb{Z}^{4} \mid 2 i+3 j+6 k+l=0\right\}
$$

as

$$
\check{e}_{1}=(2,-1,0,-1), \quad \check{e}_{2}=(3,0,-1,0), \quad \check{e}_{3}=(-1,1,0,-1),
$$

so that the Newton polytope $\Delta_{(\check{F}, \breve{G})}$ of $\check{F} / x y z w$ is the convex hull of

\begin{tabular}{c|c|c|c}
$z^{2}$ & $w^{12}$ & $x^{6}$ & $y^{2} z$ \\
\hline$(1,-1,0)$ & $(-5,1,-6)$ & $(1,1,0)$ & $(0,0,1)$
\end{tabular}

If we set

$$
\Delta:=\Delta_{(F, G)}=\{(-1,0,1),(-1,0,-1),(2,3,-1),(0,-1,0)\}
$$

then its polar dual polytope

$$
\Delta^{\circ}:=\{(1,1,0),(1,-1,0),(-5,1,-6),(-1,1,2)\}
$$

contains $\Delta_{(\breve{F}, \breve{G})}$.

\section{5 $S_{1,0}$-singularity}

The invertible polynomial $f=x^{5} y+y^{2} z+z^{2}$ is weighted homogeneous of degree $\left(q_{1}, q_{2}, q_{3} ; h\right)=$ $(3,5,10 ; 20)$. The group of maximal diagonal symmetries is given by

$$
G_{\max }(f)=\langle J(f)\rangle \text {. }
$$

The four-variable extension $F=x^{5} y+y^{2} z+z^{2}+w^{10}$ of $f$ is weighted homogeneous of weight $(3,5,10,2)$ and satisfies Assupmtion 3.1. The subgroup $G$ of $G_{\max }(F)$ is given by $G=\langle J(F)\rangle$. The lattice $M$ is given by

$$
M=\left\{(i, j, k, l) \in \mathbb{Z}^{4} \mid 3 i+5 j+10 k+2 l=0\right\} .
$$


We choose a basis of $M$ as

$$
e_{1}=(-1,-1,1,-1), \quad e_{2}=(-1,1,0,-1), \quad e_{3}=(-1,1,-1,4) .
$$

With respect this basis, the polytope $\Delta_{(F, G)}$ is the convex hull of

$$
\begin{array}{c|c|c|c}
z^{2} & w^{10} & x^{5} y & y^{2} z \\
\hline(1,0,0) & (1,-2,2) & (-2,-1,-1) & (0,1,0)
\end{array} .
$$

Similarly, we choose the basis of the group

$$
\check{M}:=\left\{(i, j, k, l) \in \mathbb{Z}^{4} \mid 2 i+4 j+3 k+l=0\right\}
$$

as

$$
\check{e}_{1}=(-2,0,1,1), \quad \check{e}_{2}=(-1,1,0,-2), \quad \check{e}_{3}=(-1,0,0,2),
$$

so that the Newton polytope $\Delta_{(\check{F}, \breve{G})}$ of $\check{F} / x y z w$ is the convex hull of

\begin{tabular}{c|c|c|c}
$y z^{2}$ & $w^{10}$ & $x^{5}$ & $x y^{2}$ \\
\hline$(1,0,-1)$ & $(-1,-1,4)$ & $(-1,-1,-1)$ & $(-1,1,1)$
\end{tabular}.

The polytope

$$
\Delta:=\{(1,0,0),(1,-2,2),(-2,-1,-1),(-1,2,0),(-2,-2,-1)\}
$$

contains $\Delta_{(F, G)}$, and its polar dual polytope

$$
\Delta^{\circ}:=\{(-1,-1,-1),(-1,1,1),(-1,1,2),(-1,-1,4),(1,0,-1),(-1,0,3)\}
$$

contains $\Delta_{(\check{F}, \breve{G})}$.

\section{6 $U_{1,0}$-singularity}

The invertible polynomial $f=x^{3} y+y^{2} z+z^{3}$ is weighted homogeneous of degree $\left(q_{1}, q_{2}, q_{3} ; h\right)=$ $(2,3,3 ; 9)$. The group of maximal diagonal symmetries is given by

$$
G_{\max }(f)=\left\langle J(f), \frac{1}{2}(1,1,0)\right\rangle \text {. }
$$

The four-variable extension $F=x^{3} y+y^{2} z+z^{3}+w^{9}$ of $f$ is weighted homogeneous of weight $(2,3,3,1)$ and satisfies Assupmtion [3.1. The subgroup $G$ of $G_{\max }(F)$ is given by $G=\left\langle J(F), \frac{1}{2}(1,1,0,0)\right\rangle$. The lattice $M$ is given by

$$
M=\left\{(i, j, k, l) \in \mathbb{Z}^{4} \mid 2 i+3 j+3 k+l=0 \text { and } i+j \equiv 0 \bmod 2\right\} .
$$

We choose a basis of $M$ as

$$
e_{1}=(1,1,0,-5), \quad e_{2}=(1,-1,0,1), \quad e_{3}=(0,0,1,-3) .
$$


With respect this basis, the polytope $\Delta_{(F, G)}$ is the convex hull of

\begin{tabular}{c|c|c|c}
$z^{3}$ & $w^{9}$ & $x^{3} y$ & $y^{2} z$ \\
\hline$(-1,0,2)$ & $(-1,0,-1)$ & $(1,1,-1)$ & $(0,-1,0)$
\end{tabular}.

Similarly, we choose the basis of the group

$$
\check{M}:=\left\{(i, j, k, l) \in \mathbb{Z}^{4} \mid 3 i+3 j+2 k+l=0\right\}
$$

as

$$
\check{e}_{1}=(1,0,-1,-1), \quad \check{e}_{2}=(1,-1,0,0), \quad \check{e}_{3}=(-1,0,2,-1),
$$

so that the Newton polytope $\Delta_{(\check{F}, \breve{G})}$ of $\check{F} / x y z w$ is the convex hull of

$$
\begin{array}{c|c|c|c}
x^{3} & w^{9} & x y^{2} & y z^{3} \\
\hline(1,1,0) & (-5,1,-3) & (1,-1,0) & (0,0,1)
\end{array} \text {. }
$$

The polytope

$$
\Delta:=\{(-1,0,2),(-1,0,-1),(1,2,-1),(1,1,-1),(0,-1,0),(0,-1,-1)\}
$$

contains $\Delta_{(F, G)}$, and its polar dual polytope

$$
\Delta^{\circ}:=\{(1,1,0),(1,-1,0),(0,0,1),(-3,0,-2),(-2,1,0),(-5,1,-3)\}
$$

contains $\Delta_{(\check{F}, \check{G})}$.

\section{7 $\quad E_{18}$-singularity}

The invertible polynomial $f=x^{5}+y^{3}+x z^{2}$ is weighted homogeneous of degree $\left(q_{1}, q_{2}, q_{3} ; h\right)=$ $(3,5,6 ; 15)$. The group of maximal diagonal symmetries is given by

$$
G_{\max }(f)=\left\langle J(f), \frac{1}{2}(0,0,1)\right\rangle .
$$

The four-variable extension $F=x^{5}+y^{3}+x z^{2}+z w^{9}$ of $f$ is weighted homogeneous of weight $(3,5,6,1)$ and satisfies Assupmtion 3.1. The subgroup $G$ of $G_{\max }(F)$ is given by $G=\left\langle J(F), \frac{1}{2}(0,0,1,1)\right\rangle$. The lattice $M$ is given by

$$
M=\left\{(i, j, k, l) \in \mathbb{Z}^{4} \mid 3 i+5 j+6 k+l=0 \text { and } k+l \equiv 0 \bmod 2\right\} .
$$

We choose a basis of $M$ as

$$
e_{1}=(1,1,0,-8), \quad e_{2}=(0,1,1,-11), \quad e_{3}=(1,0,1,-9) \text {. }
$$

With respect this basis, the polytope $\Delta_{(F, G)}$ is the convex hull of

$$
\begin{array}{c|c|c|c}
x z^{2} & w^{9} z & x^{5} & y^{3} \\
\hline(-1,0,1) & (-1,0,0) & (2,-3,2) & (1,1,-2)
\end{array} \text {. }
$$


Similarly, we choose the basis of the group

$$
\check{M}:=\left\{(i, j, k, l) \in \mathbb{Z}^{4} \mid i+3 j+4 k+l=0\right\}
$$

as

$$
\check{e}_{1}=(-1,1,-1,2), \quad \check{e}_{2}=(0,1,0,-3), \quad \check{e}_{3}=(0,-2,1,2) \text {, }
$$

so that the Newton polytope $\Delta_{(\check{F}, \check{G})}$ of $\check{F} / x y z w$ is the convex hull of

\begin{tabular}{c|c|c|c}
$z^{2} w$ & $w^{9}$ & $x^{5} z$ & $y^{3}$ \\
\hline$(1,2,2)$ & $(1,-2,0)$ & $(-4,-5,-4)$ & $(1,1,0)$
\end{tabular}.

The polytope

$$
\Delta:=\{(1,1,-2),(-1,0,0),(-1,0,1),(2,-3,2),(1,-2,1)\}
$$

contains $\Delta_{(F, G)}$, and its polar dual polytope

$$
\Delta^{\circ}:=\{(1,2,2),(1,-2,0),(-8,-11,-9),(0,1,1),(1,1,0)\}
$$

contains $\Delta_{(\check{F}, \check{G})}$.

\section{$4.8 \quad E_{19}$-singularity}

The invertible polynomial $f=x^{7}+x y^{3}+z^{2}$ is weighted homogeneous of degree $\left(q_{1}, q_{2}, q_{3} ; h\right)=$ $(2,4,7 ; 14)$. The group of maximal diagonal symmetries is given by

$$
G_{\max }(f)=\left\langle J(f), \frac{1}{3}(0,1,0)\right\rangle .
$$

The four-variable extension $F=x^{7}+x y^{3}+z^{2}+y w^{10}$ of $f$ is weighted homogeneous of weight $(2,4,7,1)$ and satisfies Assupmtion 3.1. The subgroup $G$ of $G_{\max }(F)$ is given by $G=\left\langle J(F), \frac{1}{3}(0,1,0,-1)\right\rangle$. The lattice $M$ is given by

$$
M=\left\{(i, j, k, l) \in \mathbb{Z}^{4} \mid 2 i+4 j+7 k+l=0 \text { and } j-l \equiv 0 \bmod 3\right\} .
$$

We choose a basis of $M$ as

$$
e_{1}=(3,0,0,-6), \quad e_{2}=(1,-1,0,2), \quad e_{3}=(0,1,1,-11) .
$$

With respect this basis, the polytope $\Delta_{(F, G)}$ is the convex hull of

$$
\begin{array}{c|c|c|c}
z^{2} & w^{10} y & x^{7} & x y^{3} \\
\hline(-1,2,1) & (0,-1,-1) & (2,0,-1) & (1,-3,-1)
\end{array} .
$$

Similarly, we choose the basis of the group

$$
\check{M}:=\left\{(i, j, k, l) \in \mathbb{Z}^{4} \mid i+3 j+5 k+l=0\right\}
$$


as

$$
\check{e}_{1}=(2,1,-1,0), \quad \check{e}_{2}=(0,-3,2,-1), \quad \check{e}_{3}=(-1,-1,1,-1),
$$

so that the Newton polytope $\Delta_{(\check{F}, \check{G})}$ of $\check{F} / x y z w$ is the convex hull of

$$
\begin{array}{c|c|c|c}
z^{2} & w^{10} & x^{7} y & y^{3} w \\
\hline(0,0,1) & (-6,2,-11) & (3,1,0) & (0,-1,1)
\end{array} .
$$

The polytope

$$
\Delta:=\{(-1,2,1),(0,-1,-1),(1,0,-1),(2,0,-1),(1,-3,-1)\}
$$

contains $\Delta_{(F, G)}$, and its polar dual polytope

$$
\Delta^{\circ}:=\{(0,0,1),(-6,2,-11),(4,2,-1),(1,-1,2),(0,-1,1)\}
$$

contains $\Delta_{(\check{F}, \check{G})}$.

\section{$4.9 \quad E_{20}$-singularity}

The invertible polynomial $f=x^{11}+y^{3}+z^{2}$ is weighted homogeneous of degree $\left(q_{1}, q_{2}, q_{3} ; h\right)=$ $(6,22,33 ; 66)$. The group of maximal diagonal symmetries is given by

$$
G_{\max }(f)=\langle J(f)\rangle .
$$

The four-variable extension $F=x^{11}+y^{3}+z^{2}+x w^{12}$ of $f$ is weighted homogeneous of weight $(6,22,33,5)$ and satisfies Assupmtion 3.1. The subgroup $G$ of $G_{\max }(F)$ is given by $G=\langle J(F)\rangle$. The lattice $M$ is given by

$$
M=\left\{(i, j, k, l) \in \mathbb{Z}^{4} \mid 6 i+22 j+33 k+5 l=0\right\} .
$$

We choose a basis of $M$ as

$$
e_{1}=(-1,-1,1,-1), \quad e_{2}=(0,-1,-1,11), \quad e_{3}=(2,-1,0,2) .
$$

With respect this basis, the polytope $\Delta_{(F, G)}$ is the convex hull of

$$
\begin{array}{c|c|c|c}
z^{2} & w^{12} x & x^{11} & y^{3} \\
\hline(1,0,0) & (0,1,0) & (-2,-1,4) & (-1,0,-1)
\end{array} .
$$

Similarly, we choose the basis of the group

$$
\check{M}:=\left\{(i, j, k, l) \in \mathbb{Z}^{4} \mid i+4 j+6 k+l=0\right\}
$$

as

$$
\check{e}_{1}=(-2,-1,1,0), \quad \check{e}_{2}=(-1,0,0,1), \quad \check{e}_{3}=(4,-1,0,0),
$$

so that the Newton polytope $\Delta_{(\check{F}, \breve{G})}$ of $\check{F} / x y z w$ is the convex hull of

$$
\begin{array}{c|c|c|c}
z^{2} & w^{12} & w x^{11} & y^{3} \\
\hline(1,-1,0) & (-1,11,2) & (-1,0,2) & (-1,-1,-1)
\end{array} .
$$

The polytope

$$
\Delta:=\{(1,0,0),(0,1,0),(-2,-1,4),(-1,0,-1)\}
$$

contains $\Delta_{(F, G)}$, and its polar dual polytope

$$
\Delta^{\circ}:=\{(-1,-1,-1),(-1,-1,2),(1,-1,0),(-1,11,2)\}
$$

contains $\Delta_{(\check{F}, \check{G})}$. 


\section{$4.10 \quad Z_{17}$-singularity}

The invertible polynomial $f=x^{4} y+y^{3}+x z^{2}$ is weighted homogeneous of degree $\left(q_{1}, q_{2}, q_{3} ; h\right)=$ $(2,4,5 ; 12)$. The group of maximal diagonal symmetries is given by

$$
G_{\max }(f)=\langle J(f)\rangle \text {. }
$$

The four-variable extension $F=x^{4} y+y^{3}+x z^{2}+z w^{7}$ of $f$ is weighted homogeneous of weight $(2,4,5,1 ; 12)$ and satisfies Assupmtion 3.1 . The subgroup $G$ of $G_{\max }(F)$ is given by $G=\langle J(F)\rangle$. The lattice $M$ is given by

$$
M=\left\{(i, j, k, l) \in \mathbb{Z}^{4} \mid 2 i+4 j+5 k+l=0\right\} .
$$

We choose a basis of $M$ as

$$
e_{1}=(1,1,0,-6), \quad e_{2}=(1,0,1,-7), \quad e_{3}=(0,1,1,-9) .
$$

With respect this basis, the polytope $\Delta_{(F, G)}$ is the convex hull of

$$
\begin{array}{c|c|c|c}
x z^{2} & w^{7} z & x^{4} y & y^{3} \\
\hline(-1,1,0) & (-1,0,0) & (2,1,-2) & (1,-2,1)
\end{array} .
$$

Similarly, we choose the basis of the group

$$
\check{M}:=\left\{(i, j, k, l) \in \mathbb{Z}^{4} \mid i+2 j+3 k+l=0\right\}
$$

as

$$
\check{e}_{1}=(-1,1,-1,2), \quad \check{e}_{2}=(0,-2,1,1), \quad \check{e}_{3}=(0,1,0,-2) \text {, }
$$

so that the Newton polytope $\Delta_{(\breve{F}, \breve{G})}$ of $\check{F} / x y z w$ is the convex hull of

$$
\begin{array}{c|c|c|c}
w z^{2} & w^{7} & x^{4} z & x y^{3} \\
\hline(1,2,2) & (1,0,-2) & (-3,-3,-4) & (0,-1,0)
\end{array} .
$$

The polytope

$$
\Delta:=\{(-1,0,0),(1,-2,1),(1,0,-1),(0,1,-1),(2,1,-2),(-1,1,0)\}
$$

contains $\Delta_{(F, G)}$, and its polar dual polytope

$$
\Delta^{\circ}:=\{(1,2,2),(1,1,2),(0,1,1),(1,0,-2),(1,0,1),(0,-1,0),(-6,-7,-9)\}
$$

contains $\Delta_{(\check{F}, \check{G})}$.

\section{$4.11 Z_{19}$-singularity}

The invertible polynomial $f=x^{9} y+y^{3}+z^{2}$ is weighted homogeneous of degree $\left(q_{1}, q_{2}, q_{3} ; h\right)=$ $(4,18,27 ; 54)$. The group of maximal diagonal symmetries is given by

$$
G_{\max }(f)=\langle J(f)\rangle \text {. }
$$


The four-variable extension $F=x^{9} y+y^{3}+z^{2}+x w^{10}$ of $f$ is weighted homogeneous of weight $(4,18,27,5)$ and satisfies Assupmtion 3.1. The subgroup $G$ of $G_{\max }(F)$ is given by $G=\langle J(F)\rangle$. The lattice $M$ is given by

$$
M=\left\{(i, j, k, l) \in \mathbb{Z}^{4} \mid 4 i+18 j+27 k+5 l=0\right\} .
$$

We choose a basis of $M$ as

$$
e_{1}=(-1,-1,1,-1), \quad e_{2}=(-1,2,-1,-1), \quad e_{3}=(0,-1,-1,9) .
$$

With respect this basis, the polytope $\Delta_{(F, G)}$ is the convex hull of

\begin{tabular}{c|c|c|c}
$z^{2}$ & $w^{10} x$ & $x^{9} y$ & $y^{3}$ \\
\hline$(1,0,0)$ & $(0,0,1)$ & $(-5,-3,-1)$ & $(0,1,0)$
\end{tabular}.

Similarly, we choose the basis of the group

$$
\check{M}:=\left\{(i, j, k, l) \in \mathbb{Z}^{4} \mid i+3 j+5 k+l=0\right\}
$$

as

$$
\check{e}_{1}=(-5,0,1,0), \quad \check{e}_{2}=(-3,1,0,0), \quad \check{e}_{3}=(-1,0,0,1),
$$

so that the Newton polytope $\Delta_{(\check{F}, \check{G})}$ of $\check{F} / x y z w$ is the convex hull of

$$
\begin{array}{c|c|c|c}
z^{2} & w^{10} & w x^{9} & x y^{3} \\
\hline(1,-1,-1) & (-1,-1,9) & (-1,-1,0) & (-1,2,-1)
\end{array}
$$

The polytope

$$
\Delta:=\{(1,0,0),(0,1,0),(0,0,1),(-3,-2,0),(-5,-3,-1)\}
$$

contains $\Delta_{(F, G)}$, and its polar dual polytope

$$
\Delta^{\circ}:=\{(-1,-1,-1),(-1,2,-1),(-1,2,0),(-1,-1,9),(1,-1,-1)\}
$$

contains $\Delta_{(\check{F}, \check{G})}$.

\section{$4.12 Q_{17}$-singularity}

The invertible polynomial $f=x^{5} z+x y^{3}+z^{2}$ is weighted homogeneous of degree $\left(q_{1}, q_{2}, q_{3} ; h\right)=$ $(1,3,5 ; 10)$. The group of maximal diagonal symmetries is given by

$$
G_{\max }(f)=\left\langle J(f), \frac{1}{3}(0,1,0)\right\rangle .
$$

The four-variable extension $F=x^{5} z+x y^{3}+z^{2}+y w^{7}$ of $f$ is weighted homogeneous of weight $(1,3,5,1)$ and satisfies Assupmtion 3.1. The subgroup $G$ of $G_{\max }(F)$ is given by $G=\left\langle J(F), \frac{1}{3}(0,1,0,-1)\right\rangle$. The lattice $M$ is given by

$$
M=\left\{(i, j, k, l) \in \mathbb{Z}^{4} \mid i+3 j+5 k+l=0 \text { and } j-l \equiv 0 \bmod 3\right\} .
$$


We choose a basis of $M$ as

$$
e_{1}=(3,0,0,-3), \quad e_{2}=(0,1,1,-8), \quad e_{3}=(1,0,1,-6) .
$$

With respect this basis, the polytope $\Delta_{(F, G)}$ is the convex hull of

$$
\begin{array}{c|c|c|c}
z^{2} & w^{7} y & x y^{3} & x^{5} z \\
\hline(-1,-1,2) & (0,0,-1) & (1,2,-3) & (1,-1,1)
\end{array} .
$$

Similarly, we choose the basis of the group

$$
\check{M}:=\left\{(i, j, k, l) \in \mathbb{Z}^{4} \mid i+2 j+3 k+l=0\right\}
$$

as

$$
\check{e}_{1}=(1,1,-1,0), \quad \check{e}_{2}=(-1,2,-1,0), \quad \check{e}_{3}=(1,-3,2,-1) \text {, }
$$

so that the Newton polytope $\Delta_{(\breve{F}, \breve{G})}$ of $\check{F} / x y z w$ is the convex hull of

\begin{tabular}{c|c|c|c}
$x z^{2}$ & $w^{7}$ & $x^{5} y$ & $w y^{3}$ \\
\hline$(0,1,1)$ & $(-3,-8,-6)$ & $(3,0,1)$ & $(0,1,0)$
\end{tabular}.

The polytope

$$
\Delta:=\{(-1,-1,2),(0,0,-1),(0,-1,0),(3,-1,0),(1,2,-3)\}
$$

contains $\Delta_{(F, G)}$, and its polar dual polytope

$$
\Delta^{\circ}:=\{(0,1,1),(-3,-8,-6),(4,-1,1),(2,1,1),(0,1,0)\}
$$

contains $\Delta_{(\check{F}, \check{G})}$.

\subsection{3 $Q_{18}$-singularity}

The invertible polynomial $f=x^{8} z+y^{3}+z^{2}$ is weighted homogeneous of degree $\left(q_{1}, q_{2}, q_{3} ; h\right)=$ $(3,16,24 ; 48)$. The group of maximal diagonal symmetries is given by

$$
G_{\max }(f)=\langle J(f)\rangle \text {. }
$$

The four-variable extension $F=x^{8} z+y^{3}+z^{2}+x w^{9}$ of $f$ is weighted homogeneous of weight $(3,16,24,5)$ and satisfies Assupmtion 3.1. The subgroup $G$ of $G_{\max }(F)$ is given by $G=\langle J(F)\rangle$. The lattice $M$ is given by

$$
M=\left\{(i, j, k, l) \in \mathbb{Z}^{4} \mid 3 i+16 j+24 k+5 l=0\right\} .
$$

We choose a basis of $M$ as

$$
e_{1}=(-1,-1,1,-1), \quad e_{2}=(-1,2,-1,-1), \quad e_{3}=(0,-1,-1,8) .
$$


With respect this basis, the polytope $\Delta_{(F, G)}$ is the convex hull of

\begin{tabular}{c|c|c|c}
$z^{2}$ & $w^{9} x$ & $x^{8} z$ & $y^{3}$ \\
\hline$(1,0,0)$ & $(0,0,1)$ & $(-4,-3,-1)$ & $(0,1,0)$
\end{tabular}.

Similarly, we choose the basis of the group

$$
\check{M}:=\left\{(i, j, k, l) \in \mathbb{Z}^{4} \mid i+3 j+4 k+l=0\right\}
$$

as

$$
\check{e}_{1}=(-4,0,1,0), \quad \check{e}_{2}=(-3,1,0,0), \quad \check{e}_{3}=(-1,0,0,1),
$$

so that the Newton polytope $\Delta_{(\check{F}, \breve{G})}$ of $\check{F} / x y z w$ is the convex hull of

$$
\begin{array}{c|c|c|c}
x z^{2} & w^{9} & w x^{8} & y^{3} \\
\hline(1,-1,-1) & (-1,-1,8) & (-1,-1,0) & (-1,2,-1)
\end{array} .
$$

The polytope

$$
\Delta:=\{(1,0,0),(0,1,0),(0,0,1),(-3,-2,0),(-4,-3,-1)\}
$$

contains $\Delta_{(F, G)}$, and its polar dual polytope

$$
\Delta^{\circ}:=\{(-1,-1,-1),(1,-1,-1),(1,-1,0),(-1,-1,8),(-1,2,-1)\}
$$

contains $\Delta_{(\check{F}, \check{G})}$.

\subsection{4 $W_{18}$-singularity}

The invertible polynomial $f=x^{7}+y^{2} z+z^{2}$ is weighted homogeneous of degree $\left(q_{1}, q_{2}, q_{3} ; h\right)=$ $(4,7,14 ; 28)$. The group of maximal diagonal symmetries is given by

$$
G_{\max }(f)=\langle J(f)\rangle \text {. }
$$

The four-variable extension $F=x^{7}+y^{2} z+z^{2}+x w^{8}$ of $f$ is weighted homogeneous of weight $(4,7,14,3)$ and satisfies Assupmtion 3.1. The subgroup $G$ of $G_{\max }(F)$ is given by $G=\langle J(F)\rangle$. The lattice $M$ is given by

$$
M=\left\{(i, j, k, l) \in \mathbb{Z}^{4} \mid 4 i+7 j+14 k+3 l=0\right\} .
$$

We choose a basis of $M$ as

$$
e_{1}=(0,2,-1,0), \quad e_{2}=(1,1,-1,1), \quad e_{3}=(-1,0,-1,6) .
$$

With respect this basis, the polytope $\Delta_{(F, G)}$ is the convex hull of

$$
\begin{array}{c|c|c|c}
z^{2} & w^{8} x & x^{7} & y^{2} z \\
\hline(0,-1,0) & (-1,1,1) & (-3,5,-1) & (1,-1,0)
\end{array} .
$$


Similarly, we choose the basis of the group

$$
\check{M}:=\left\{(i, j, k, l) \in \mathbb{Z}^{4} \mid i+4 j+2 k+l=0\right\}
$$

as

$$
\check{e}_{1}=(-3,1,0,-1), \quad \check{e}_{2}=(5,-1,-1,1), \quad \check{e}_{3}=(-1,0,0,1),
$$

so that the Newton polytope $\Delta_{(\check{F}, \breve{G})}$ of $\check{F} / x y z w$ is the convex hull of

$$
\begin{array}{c|c|c|c}
y z^{2} & w^{8} & w x^{7} & y^{2} \\
\hline(-1,-1,-1) & (0,1,6) & (0,1,-1) & (2,1,0)
\end{array} .
$$

The polytope

$$
\Delta:=\{(0,-1,0),(-1,1,1),(-3,5,-1),(2,-1,0),(0,0,1)\}
$$

contains $\Delta_{(F, G)}$, and its polar dual polytope

$$
\Delta^{\circ}:=\{(-1,-1,-1),(0,1,6),(0,1,-1),(1,1,-1),(2,1,0)\}
$$

contains $\Delta_{(\check{F}, \check{G})}$.

\subsection{5 $S_{17}$-singularity}

The invertible polynomial $f=x^{6} y+y^{2} z+z^{2}$ is weighted homogeneous of degree $\left(q_{1}, q_{2}, q_{3} ; h\right)=$ $(1,2,4 ; 8)$. The group of maximal diagonal symmetries is given by

$$
G_{\max }(f)=\left\langle J(f), \frac{1}{3}(1,0,0)\right\rangle .
$$

The four-variable extension $F=x^{6} y+y^{2} z+z^{2}+x w^{7}$ of $f$ is weighted homogeneous of weight $(1,2,4,1)$ and satisfies Assupmtion 3.1. The subgroup $G$ of $G_{\max }(F)$ is given by $G=\left\langle J(F), \frac{1}{3}(1,0,0,-1)\right\rangle$. The lattice $M$ is given by

$$
M=\left\{(i, j, k, l) \in \mathbb{Z}^{4} \mid i+2 j+4 k+l=0 \text { and } i-l \equiv 0 \bmod 3\right\} .
$$

We choose a basis of $M$ as

$$
e_{1}=(1,-1,0,1), \quad e_{2}=(0,1,1,-6), \quad e_{3}=(0,0,3,-12) .
$$

With respect this basis, the polytope $\Delta_{(F, G)}$ is the convex hull of

$$
\begin{array}{c|c|c|c}
z^{2} & w^{7} x & x^{6} y & y^{2} z \\
\hline(-1,-2,1) & (0,-1,0) & (5,5,-2) & (-1,0,0)
\end{array} .
$$

Similarly, we choose the basis of the group

$$
\check{M}:=\left\{(i, j, k, l) \in \mathbb{Z}^{4} \mid i+3 j+2 k+l=0\right\}
$$


as

$$
\check{e}_{1}=(5,-1,-1,0), \quad \check{e}_{2}=(5,0,-2,-1), \quad \check{e}_{3}=(-2,0,1,0),
$$

so that the Newton polytope $\Delta_{(\breve{F}, \breve{G})}$ of $\check{F} / x y z w$ is the convex hull of

\begin{tabular}{c|c|c|c}
$y z^{2}$ & $w^{7}$ & $w x^{6}$ & $x y^{2}$ \\
\hline$(0,1,3)$ & $(1,-6,-12)$ & $(1,0,0)$ & $(-1,1,0)$
\end{tabular}.

The polytope

$$
\Delta:=\{(-1,-2,1),(0,-1,0),(6,5,-2),(5,5,-2),(-1,2,-1)\}
$$

contains $\Delta_{(F, G)}$, and its polar dual polytope

$$
\Delta^{\circ}:=\{(0,1,3),(1,-6,-12),(1,1,2),(-1,1,0),(0,-3,-7)\}
$$

contains $\Delta_{(\check{F}, \check{G})}$.

\section{$4.16 \quad U_{16}$-singularity}

The invertible polynomial $f=x^{5}+y^{2} z+y z^{2}$ is weighted homogeneous of degree $\left(q_{1}, q_{2}, q_{3} ; h\right)=$ $(3,5,5 ; 15)$. The group of maximal diagonal symmetries is given by

$$
G_{\max }(f)=\langle J(f)\rangle \text {. }
$$

The four-variable extension $F=x^{5}+y^{2} z+y z^{2}+x w^{6}$ of $f$ is weighted homogeneous of weight $(3,5,5,2)$ and satisfies Assupmtion 3.1. The subgroup $G$ of $G_{\max }(F)$ is given by $G=\langle J(F)\rangle$. The lattice $M$ is given by

$$
M=\left\{(i, j, k, l) \in \mathbb{Z}^{4} \mid 3 i+5 j+5 k+2 l=0\right\} .
$$

We choose a basis of $M$ as

$$
e_{1}=(-1,-1,0,4), \quad e_{2}=(-1,0,-1,4), \quad e_{3}=(0,-1,-1,5) .
$$

With respect this basis, the polytope $\Delta_{(F, G)}$ is the convex hull of

$$
\begin{array}{c|c|c|c}
y z^{2} & w^{6} x & x^{5} & y^{2} z \\
\hline(1,0,-1) & (0,0,1) & (-2,-2,3) & (0,1,-1)
\end{array} .
$$

Similarly, we choose the basis of the group

$$
\check{M}:=\left\{(i, j, k, l) \in \mathbb{Z}^{4} \mid i+2 j+2 k+l=0\right\}
$$

as

$$
\check{e}_{1}=(-2,0,1,0), \quad \check{e}_{2}=(-2,1,0,0), \quad \check{e}_{3}=(3,-1,-1,1),
$$

so that the Newton polytope $\Delta_{(\check{F}, \breve{G})}$ of $\check{F} / x y z w$ is the convex hull of

\begin{tabular}{c|c|c|c}
$y z^{2}$ & $w^{6}$ & $w x^{5}$ & $y^{2} z$ \\
\hline$(0,-1,-1)$ & $(4,4,5)$ & $(-1,-1,0)$ & $(-1,0,-1)$
\end{tabular}.

The polytope

$$
\Delta:=\{(1,0,-1),(0,0,1),(-2,-2,3),(0,1,-1)\}
$$

contains $\Delta_{(F, G)}$, and its polar dual polytope

$$
\Delta^{\circ}:=\{(1,-2,-1),(4,4,5),(-2,-2,-1),(-2,1,-1)\}
$$

contains $\Delta_{(\check{F}, \breve{G})}$. 


\section{References}

[Arn75] V. I. Arnol'd, Critical points of smooth functions, and their normal forms, Uspehi Mat. Nauk 30 (1975), no. 5(185), 3-65. MR 0420689 (54 \#8701)

[Arn76] _ Local normal forms of functions, Invent. Math. 35 (1976), 87-109. MR 0467795 (57 \#7646)

[Bat94] Victor V. Batyrev, Dual polyhedra and mirror symmetry for Calabi-Yau hypersurfaces in toric varieties, J. Algebraic Geom. 3 (1994), no. 3, 493-535. MR MR1269718 (95c:14046)

[BH93] Per Berglund and Tristan Hübsch, A generalized construction of mirror manifolds, Nuclear Phys. B 393 (1993), no. 1-2, 377-391. MR MR1214325 (94k:14031)

[Buc87] Ragnar-Olaf Buchweitz, Maximal Cohen-Macaulay modules and Tate-cohomology over Gorenstein rings, Available from https://tspace.library.utoronto.ca/handle/1807/16682, 1987.

[Eis80] David Eisenbud, Homological algebra on a complete intersection, with an application to group representations, Trans. Amer. Math. Soc. 260 (1980), no. 1, 35-64. MR MR570778 (82d:13013)

[EP13] Wolfgang Ebeling and David Ploog, A geometric construction of CoxeterDynkin diagrams of bimodal singularities, Manuscripta Math. 140 (2013), no. 1-2, 195-212. MR 3016490

[FU11] Masahiro Futaki and Kazushi Ueda, Homological mirror symmetry for Brieskorn-Pham singularities, Selecta Math. (N.S.) 17 (2011), no. 2, 435-452.

[FU13] _ Homological mirror symmetry for singularities of type D, Math. Z. 273 (2013), no. 3-4, 633-652. MR 3030671

[GVW89] B. R. Greene, C. Vafa, and N. P. Warner, Calabi-Yau manifolds and renormalization group flows, Nuclear Phys. B 324 (1989), no. 2, 371-390. MR 1025421 (91c:14042)

[Kob08] Masanori Kobayashi, Duality of weights, mirror symmetry and Arnold's strange duality, Tokyo J. Math. 31 (2008), no. 1, 225-251. MR 2426805 (2010f:32022)

[Kon95] Maxim Kontsevich, Homological algebra of mirror symmetry, Proceedings of the International Congress of Mathematicians, Vol. 1, 2 (Zürich, 1994) (Basel), Birkhäuser, 1995, pp. 120-139. MR MR1403918 (97f:32040)

[Kra] Marc Krawitz, FJRW rings and Landau-Ginzburg mirror symmetry, arXiv:0906.0796.

[Mar90] Emil J. Martinec, Criticality, catastrophes, and compactifications, Physics and mathematics of strings, World Sci. Publ., Teaneck, NJ, 1990, pp. 389-433. MR 1104265 (93d:32058) 
[Orl04] D. O. Orlov, Triangulated categories of singularities and D-branes in LandauGinzburg models, Tr. Mat. Inst. Steklova 246 (2004), no. Algebr. Geom. Metody, Svyazi i Prilozh., 240-262. MR MR2101296

[Or109] Dmitri Orlov, Derived categories of coherent sheaves and triangulated categories of singularities, Algebra, arithmetic, and geometry: in honor of Yu. I. Manin. Vol. II, Progr. Math., vol. 270, Birkhäuser Boston Inc., Boston, MA, 2009, pp. 503-531. MR 2641200 (2011c:14050)

[Sei08] Paul Seidel, Fukaya categories and Picard-Lefschetz theory, Zurich Lectures in Advanced Mathematics, European Mathematical Society (EMS), Zürich, 2008. MR MR2441780

[Sei10] - Suspending Lefschetz fibrations, with an application to local mirror symmetry, Comm. Math. Phys. 297 (2010), no. 2, 515-528. MR 2651908

[Tak09] Atsushi Takahashi, Talk at Workshop on Homological Mirror Symmetry and Related Topics, University of Miami, slides available at http://math. berkeley.edu/ auroux/frg/miami09.html, 2009.

[Ued] Kazushi Ueda, Hyperplane sections and stable derived categories, to appear in Proc. Amer. Math. Soc., arXiv:1207.1167.

[VW89] Cumrun Vafa and Nicholas Warner, Catastrophes and the classification of conformal theories, Phys. Lett. B 218 (1989), no. 1, 51-58. MR 983349 (90m:81135)

[Wit93] Edward Witten, Phases of $N=2$ theories in two dimensions, Nuclear Phys. B 403 (1993), no. 1-2, 159-222. MR MR1232617 (95a:81261)

Makiko Mase

Department of Mathematics and Information Sciences, Tokyo Metropolitan University, 1-1 Minami-Osawa, Hachioji-shi Tokyo, 192-0397, Japan.

Advanced Mathematical Institute, Osaka City University, 3-3-138 Sugimoto, Sumiyoshiku, Osaka, 558-8585, Japan.

e-mail address : mtmase@arion.ocn.ne.jp

Kazushi Ueda

Department of Mathematics, Graduate School of Science, Osaka University, Machikaneyama 1-1, Toyonaka, Osaka, 560-0043, Japan.

e-mail address : kazushi@math.sci.osaka-u.ac.jp 